\title{
Transcriptional signature of TP53 biallelic inactivation identifies a group of multiple myeloma patients without this genetic condition but with dismal outcome.
}

\section{Norma C. Gutiérrez ( $\nabla$ normagu@usal.es)}

Hematology Department, University Hospital of Salamanca, IBSAL, Salamanca

Cristina De Ramón

Hematology Department, University Hospital of Salamanca, IBSAL, Salamanca

Elizabeta A. Rojas

Hematology Department, University Hospital of Salamanca, IBSAL, Salamanca Igancio J. Cardona-Benavides

Hematology Department, University Hospital of Salamanca, IBSAL, Salamanca

Maria Victoria Mateos

Hematology Department, University Hospital of Salamanca, IBSAL, Salamanca

Luis A. Corchete

Hematology Department, University Hospital of Salamanca, IBSAL, Salamanca

\section{Research Article}

Keywords: TP53 biallelic inactivation, Double-hit TP53, Multiple myeloma

Posted Date: February 23rd, 2022

DOI: https://doi.org/10.21203/rs.3.rs-1380448/v1

License: (c) (i) This work is licensed under a Creative Commons Attribution 4.0 International License.

Read Full License 


\section{Abstract}

Background: Biallelic inactivation of TP53 has been included in the definition of double-hit (DH) multiple myeloma (MM), which entails an ominous prognosis even when treated with the most innovative drugs. However, this condition, or even the presence of high-risk cytogenetic abnormalities, cannot accurately capture the $15-20 \%$ of the MM population with a median overall survival below 24 months. This prompted us to look for other MM patients who might have transcriptional characteristics similar to those with DH-TP53.

Methods: We analyzed RNA-seq, whole-genome and whole-exome sequencing data from 660 newly diagnosed MM (NDMM) patients from the MMRF (Multiple Myeloma Research Foundation) CoMMpass study to characterize the transcriptional signature of DH-TP53 MM. This gene signature was used to build a score based on a Spearman correlation coefficient and a scaled GINI index. Two other gene expression series from GEO (GSE4581 and GSE136400) comprising a total of 850 patients were used as validation cohorts.

Results: We found 78 genes that were exclusively deregulated in DH-TP53 patients. A score based on these genes identified a group of 50 patients who shared the same transcriptional profile (DH-TP53-like group) whose prognosis was particularly unfavorable (median overall survival [OS] $<2$ years), despite not harboring the biallelic inactivation of TP53. Patients included in the DH-TP53-like group had significantly shorter OS and progression-free survival (PFS) than those without the DH-TP53 gene expression profile (hazard ratio [HR] 4.07 [95\% Cl, 2.76-5.98], adjusted $p<0.001$, and $\mathrm{HR} 3.44$ [95\% Cl, 2.46-4.81], adjusted $p$ $<0.001$, respectively). The prognostic value of the DH-TP53 score was externally validated using gene expression data obtained by microarray analysis. Furthermore, our DH-TP53 score refined the traditional prognostic stratification of MM patients according to the cytogenetic abnormalities and International Staging System (ISS).

Conclusions: The expression signature of DH-TP53 MM was shared by other MM patients without TP53 biallelic inactivation (DH-TP53-like group). The DH-TP53 score could be a useful tool for identifying ultrahigh-risk MM patients without concomitant del(17p) and TP53 mutations.

\section{Background}

Multiple myeloma (MM) is a B-cell neoplasm characterized by great genomic and molecular complexity that largely explains the variability observed during the clinical course and in treatment response [1]. Cytogenetic abnormalities remain the most relevant prognostic factors. Deletion of chromosome 17p [del (17p)], which contains the TP53 locus, is present in $8-13 \%$ of newly diagnosed MM (NDMM) patients and is probably the cytogenetic alteration associated with the most unfavorable prognosis [2]. TP53 mutations, which also have a negative impact on survival, are also uncommon, occurring in only $3-6 \%$ of NDMM patients [3, 4]. TP53 mutations are more frequent in patients with del(17p) [5]. Biallelic 
inactivation of TP53 observed when the two abnormalities are present together, has been included in the definition of double-hit (DH) MM. DH-TP53, although rarely seen, entails an ominous prognosis [6].

Moreover, p53 can be deregulated by other mechanisms different from changes in DNA gene sequence, such as epigenetic regulation or altered expression of its regulators, which eventually lead to p53 dysfunction. The functionality of the different pathways controlled by $\mathrm{p} 53$ could be inferred by the expression of their numerous target genes. In this regard, a signature based on three genes (PUMA, GADD45 and THBS1) has been described to be associated with TP53 status in MM [7]. Furthermore, a transcriptional signature of patients with biallelic inactivation of TP53 has recently been defined. Interestingly, some relevant pathways deregulated in this signature, such as those related to cell cycle control and $M Y C$ regulation were also altered in the expression signatures specific to biallelic events involving other tumor suppressor genes [8].

Compared with solid tumors, DH-TP53 in MM is much more infrequent. However, ultra-high-risk MM, defined as those patients with a median overall survival less than 24 months, represents $15-20 \%$ of the MM population [9]. Although other high-risk cytogenetic abnormalities may account for this adverse prognosis, these kind of cytogenetic alterations are not present in all patients with such an unfavorable outcome. This prompted us to define the transcriptional signature of DH-TP53 and to find out if it was present in other patients who did not have biallelic inactivation of TP53.

\section{Methods}

\section{Patient cohorts.}

Genomic and transcriptomic data of 660 NDMM patients from the Multiple Myeloma Research Fundation (MMRF) CoMMpass study (NTC014554297, https://research.themmrf.org/) were analyzed. Survival data were retrieved from the interim analysis 16 release. The characteristics of the population are described in Additional file 1: Table S1. In addition to the CoMMpass database, two other series of MM patients, which included data from gene expression profiling analyzed by Affymetrix Human Genome U133 Plus 2.0 microarray, were selected as validation cohorts. One of them contained 414 patients treated according to total therapy 2 (256 cases) and total therapy 3 (158 patients) studies (GSE4581), and the other comprised 436 NDMM patients (GSE136400: only the CD138-positive cell samples were used for the analysis) enrolled in the total therapy $3-5$ studies.

\section{Identification of DH- TP53 signature.}

Information about $/ G H$ translocations $[t(4 ; 14), t(11 ; 14)$ and $t(14 ; 16)]$ and copy number abnormalities [1q gain and del(17p)] were obtained from the whole-genome sequencing-based fluorescent in situ hybridization (Seq-FISH) data [10]. Non-synonymous TP53 mutations assessed by whole-exome sequencing were also included in the analysis. Based on these chromosomal and gene alterations, six groups and a normal FISH group were defined. RNA-seq gene expression in the six groups was compared 
with the normal FISH group to generate the corresponding differentially expressed gene lists (falsediscovery rate $(\mathrm{FDR})<0.05)$. All contrasts were performed using the DESeq2 R package (version 1.30.1) on HTSeq count data. Genes from the six lists were then gathered to derive a Venn diagram using the ggplot2 R package (version 3.3.5), which allowed us to determine the exclusive DH-TP53 signature.

\section{Score generation.}

The DH-TP53 signature was used to build a score [11]. Briefly, we generated two gene expression matrices normalizing RNA-seq data using the vst function of the DESeq2 package or the cpm function from the limma package (version 3.46.0). We then calculated a Spearman correlation coefficient using the psych package (version 2.1.6), and a scaled GINI index through a random forest approach using the h2o R package (version 3.32.1.3) for both matrices to identify the genes that best discriminated between DH-TP53 from normal FISH. The score was then calculated as the mean of the absolute values of these four parameters multiplied by the sign of the correlation coefficient. All the genes for which the $95 \%$ confidence interval of the score included zero were discarded. Finally, we applied the score for each gene $g$ and calculated the sum for each patient $p$ using the following formula:

$$
\text { DH }- \text { TP53 } 5 \text { score }_{p}=\sum_{g}\left(\text { score }_{g} * \log _{2} \text { expression }_{g p}\right)
$$

The score values for each gene $g$ are listed in Additional file 2: Table S1. We used the value of the first quartile of the score in the DH-TP53 group as the cut-off to determine the DH-TP53-like group.

\section{Validation of DH-}

TP53 score. To validate the performance of the DH-TP53 score, we processed data from the aforementioned two GEO series [12]. Thus, raw data from the GSE136400 series [13] were backgroundsubtracted, quantile-normalized and $\log _{2}$-transformed using the affy package (version 1.68 .0 ) in $\mathrm{R}$ with a custom reference for the Human Genome U133 Plus 2.0 array from BrainArray (Ensembl version 25.0.0) [14]. As no raw data were available from GSE4581 [15], we used the previously pre-processed data from

the GEO website. We transformed the probeset identifiers from the Human Genome U133 plus 2.0 array to Ensembl identifiers to match RNA-seq data, and duplicated genes were randomly removed. It should be noted that this second approach is less precise than the BrainArray approach, as microarray probe ambiguity is not taken into account [16]. The per-patient score was then calculated for the two validation series using the gene weights obtained in the main CoMMpass cohort. Patient scores from these two series were arranged in descending order and split into two groups, based on a moving window of $5 \%$ of the patients, to enable the survival analysis to be carried out. 


\section{Statistical analysis.}

Univariable survival analysis was performed in R through the survival (version 3.2-7), survminer (version 0.4.9) and ggplot2 packages using the Kaplan-Meier estimator, with progression-free survival (PFS) and overall survival (OS) as endpoints. The resulting survival curves were compared using the log-rank test. Multivariable Cox proportional-hazards models were fitted in $\mathrm{R}$ through the coxph function of the survival package. The multicollinearity among predictor variables was quantified using variance inflation factors with the car package (version 3.0-12). The proportional-hazards assumption of these models was evaluated through the evaluation of the Schoenfeld residuals in R. Forest plots for multivariable analysis were drawn using the forestmodel package (version 0.6.2). Group differences between categorical variables were assessed with the chi square and Fisher's exact tests, depending on the number of patients included in each group; those for continuous variables were examined using the Mann-Whitney $\mathrm{U}$ and Kruskal-Wallis tests, depending on the number of contrasted groups. Post-hoc tests for chi-square and Fisher's exact tests were performed using the fifer package (version 1.1). The Benjamini-Hochberg method was applied to adjust the $p$-value $(p)$ for multiple testing, as required. The threshold of significance for unadjusted and adjusted values of $p$ was set to 0.05 . These analyses were performed using IBM SPSS Statistics 26.0 and R (version 4.0.4) packages.

\section{Results}

\section{Identification of transcriptional pattern associated with TP53 biallelic inactivation}

The 660-patient cohort was divided into six groups based on their cytogenetic and TP53 mutation status: $t(4 ; 14), t(11 ; 14), t(14 ; 16), 1 q$ gain, del(17p) and DH-TP53. Gene expression profiles of these groups were determined by contrasting their RNA-seq data against those of patients with normal FISH (considered as the cases with none of the aforementioned abnormalities). Differential expression analyses of the 23 patients harboring TP53 biallelic inactivation (DH-TP53 group) identified 78 genes (FDR < 0.05$)$ after subtracting the gene expression signatures of the five other groups (Fig. 1). The interactions between the proteins encoded by the genes involved in the DH-TP53 signature were represented using a weighted functional association network in STRING [17] (Additional file 2: Figure S1).

\section{Development of DH- TP53 score}

All the 78 genes exclusively deregulated in the DH-TP53 group met the inclusion criteria to be part of the DH-TP53 score (Fig. 2A). Based on this, we assigned the patients with a score above the first quartile of the DH-TP53 group to a new subgroup named DH-TP53-like. This subgroup consisted of 50 out of 660 (7.5\%) NDMM patients and did not harbor the combination of the del(17p) and TP53 mutation. The other $587 \mathrm{MM}$ cases corresponded with those patients who did not present biallelic inactivation of TP53 or whose scores were less than the selected threshold ("other patients" group) (Fig. 2B). 


\section{Clinical and biological characteristics of the DH- TP53 -like group}

No statistically significant differences in age, gender and Eastern Cooperative Oncology Group (ECOG) performance status were found between the DH-TP53 and DH-TP53-like groups defined by the score, or between them and the other patients. On the contrary, we detected higher levels of leucocytes, creatinine, beta-2-microglobulin, C-reactive protein and lactate dehydrogenase in the DH-TP53-like group than in the "other patients" group ( $p<0.05$, Additional file 1: Table S1).

Considering the most frequent cytogenetic abnormalities observed in $M M$, we found that $t(14 ; 16)$ was present in $12 \%(6 / 50)$ of the DH-TP53-like patients compared with $3 \%(20 / 587)$ of cases included in the "other patients" group (post-hoc adjusted $p=0.035$ ). 1q gain was significantly more frequent in the $\mathrm{DH}$ TP53-like group than in the other patients in the population, including those of the DH-TP53 group ( $p=$ 0.001). It is noteworthy that no statistically significant differences in the proportion of patients with del(17p) were observed between the DH-TP53-like and the "other patients" groups. The other cytogenetic abnormalities were equally distributed across the DH-TP53, the DH-TP53-like and the "other patients" groups (Fig. 3A; Additional file 1: Table S1).

We next analyzed the distribution of the most prevalent mutated genes across the three groups. None of the DH-TP53-like patients had any TP53 non-synonymous mutation, although $4 \%(2 / 50)$ of them harbored other types of TP53 mutations. Conversely, the DH-TP53-like group was enriched for MAX mutations relative to the "other patients" group (post-hoc adjusted $p=0.028$ ). We also noticed that none of the DH-TP53 patients had mutations of the NRAS gene (Fig. 3B).

\section{Prognostic value of DH- TP53 score}

DH-TP53 originated by the combination of the del(17p) and TP53 mutations had a significantly negative impact on survival (Fig. 4). On the other hand, the DH-TP53-like group defined by the score had a significantly shorter OS (hazard ratio [HR] 4.07 [95\% Cl, 2.76-5.98], adjusted $p<0.001$ ) and PFS (HR 3.44 [95\% Cl, 2.46-4.81], adjusted $p<0.001$ ) than those without this DH-TP53 gene expression pattern (Fig. 4). The PFS for DH-TP53-like group was even worse than that described for the DH-TP53 group (HR 1.83 [95\% Cl, 1.00-3.35], $p=0.046$; Fig. 4B).

In order to gain more insight into the prognostic value of the TP53 score, we carried out a survival analysis for the most frequent cytogenetic abnormalities, 1q gain, $\mathrm{t}(11 ; 14), \mathrm{t}(4 ; 14)$ and del(17p), stratifying samples according to whether they belonged to DH-TP53-like group or not. We observed that survival for any of the cytogenetic abnormalities was significantly shortened in the DH-TP53-like group ( $p$ $<0.05$ ) (Fig. 5).

We also investigated the impact of the DH-TP53-like group on the risk stratification according to International Staging System (ISS). The DH-TP53-like group combined with ISS III had a median PFS and 
OS of 11 and 17 months, respectively, whereas for the rest of patients with ISS III median PFS and OS were of 28 and 59 months, respectively $(p<0.001)$. Likewise, median PFS and OS of patients with ISS I/II were reduced from 47 and 95 months to 19 and 50 months, respectively, $(p<0.001)$ when the patients belonged to DH-TP53-like group (Fig. 6).

We performed a multivariable Cox model including the DH-TP53-like group defined by our score, the age at diagnosis and the well-established high-risk prognostic factors in MM, ISS, high-risk cytogenetic abnormalities and DH-TP53 group. The DH-TP53-like group was selected as an independent factor for PFS with the highest HR (HR 3.84 [95\% $\mathrm{Cl} 2.51-5.88]$; $p<0.001)$ along with DH-TP53 group, age, ISS stage III and 1q gain. The negative impact of DH-TP53-like group was also maintained in the Cox model for OS (HR 3.32 [95\% Cl, 2.31-4.77]; p<0.001) (Fig. 7).

\section{Validation of the DH- TP53 score}

Finally, we externally validated the DH-TP53 score in two previously published series: GSE4581 $(n=414)$ and GSE136400 $(n=436)$. Both series included microarray gene expression data from NDMM patients. Using these microarray data, we identified 68 and 63 out of the 78 genes of the DH-TP53 score, respectively, which were then used to calculate the score. The survival analysis of these two series clearly showed two curves whose separation widened as the cut-off value decreased. Thus, patients with a higher score, between $5-25 \%$ of the validation cohort, in both datasets showed a particularly adverse prognosis compared with the other patients ( $p<0.001$; Additional file 3: Figure S1).

\section{Discussion}

TP53 abnormalities, although uncommon at the time of diagnosis, remain one of the most important prognostic factors in MM patients, specially when del(17p) and TP53 mutation are present together. p53 might be inactivated by other mechanisms, as has been described in other lymphoid malignancies [18]. In order to ascertain this possibility, we searched for the presence of the transcriptional signature associated with DH-TP53 in NDMM patients who did not present these DNA alterations. Expression signatures of TP53 status have been defined in solid tumors $[19,20]$. In MM, previous studies have identified differentially expressed genes related to TP53 expression [21] and a p53 target gene signature associated with TP53 gene aberrations [7]. Recently, the impact of the biallelic inactivation of TP53 on gene expression has been analyzed [8]. The novelty of our study is to demonstrate that there are MM patients with very unfavorable prognosis, which have a transcriptional signature like that of patients with $\mathrm{DH}$ TP53, but without having these TP53 abnormalities. Strikingly, this DH-TP53-like group showed even shorter PFS than DH-TP53 patients considered as ultra-high-risk patients [6].

It should be noted that $60 \%$ of patients belonging to DH-TP53-like group were classified into ISS stage III, compared to approximately $30 \%$ of DH-TP53 and "other patients" groups. This reflects the high tumor mass and renal function impairment observed in DH-TP53-like patients and highlights the validity of ISS 
in discriminating high-risk patients [22], even after the introduction of novel agents. Nonetheless, in the multivariable Cox model, the DH-TP53-like group maintained its independent negative impact on survival.

Cytogenetic alterations do not seem to be the cause of the poor outcomes associated with DH-TP53-like group, as the distribution of International Myeloma Working Group (IMWG)-defined high-risk cytogenetic abnormalities [23] was similar to that found in the "other patients" group. In fact, the adverse prognosis of the patients included in the DH-TP53-like group was not properly captured by the revised-ISS (R-ISS) [24], since up to $67 \%$ of DH-TP53-like patients were not classified as high-risk patients. On the other hand, it is noteworthy that patients with 1q gain were over-represented in DH-TP53-like group. In fact, 1q gain was identified as an independent prognostic factor in the multivariable analysis, as has recently been reported in the analysis baseline cohort of the CoMMpass study [25].

More importantly, the combination of DH-TP53 like group and ISS stage significantly improved prediction of outcome in MM. The median OS dropped from 59 months for patients with ISS III to 17 months for patients with ISS III that belonged to DH-TP53 like group.

Despite del(17p) is one of the most consistent high-risk cytogenetic abnormalities included in the main risk stratification models, its negative impact is not observed in some MM patients. In this context, different approaches, such as higher cut-off value for del(17p) [26] and biallelic inactivation of the TP53 gene [6], have been published in an attempt to distinguish those patients with del(17p) who have extremely poor outcome. One of the contributions of this work in this regard is that among the patients with del(17p) it is possible to identify a subgroup without TP53 mutations that have a prognosis as poor as those patients with TP53 biallelic inactivation. Furthermore, our DH-TP53 score refined the prognostic value not only of other cytogenetic abnormalities associated with poor outcome, such as 1q gain and $t(4 ; 14)$, but also of $t(11 ; 14)$, which has been usually linked to favorable outcomes in MM. Thus, patients with $1 \mathrm{q}$ gain, $\mathrm{t}(4 ; 14)$ or $\mathrm{t}(11 ; 14)$ who simultaneously belonged to $\mathrm{DH}$-TP53-like group had significantly shorter PFS and OS.

An interesting finding emerged when the frequency of the most common point mutations in MM was assessed according to the presence of DH-TP53 signature. A significantly greater proportion of MAX gene mutations was observed in DH-TP53-like patients. Loss of function of MAX in MM patients has been associated with the proliferative subtype [25]. The enrichment of MAX mutations in DH-TP53-like patients, who experience a particular poor outcome, deserves to be investigated extensively.

\section{Conclusions}

In summary, the DH-TP53 and DH-TP53-like groups share the same transcriptional signature, although the biallelic inactivation of TP53 is only present in the former group. There are at least two possible explanations for this finding. One is that the activity of p53 is attenuated by mechanisms other than DNA alterations, like overexpression of negative regulators; the other explanation is that the combination of other genetic alterations leads to the deregulation of transcriptional pathways that can be superimposed on those deregulated by the biallelic inactivation of TP53 $[27,28]$. Hence, the DH-TP53 score described in 
this study could be a useful tool for identifying MM patients with p53 dysfunction, bearing in mind that a portion of these patients are not evidenced through FISH and DNA sequencing analysis.

\section{Abbreviations}

- del(17p): deletion of chromosome 17p

- DH: double-hit

- DH-TP53: double-hit TP53

- ECOG: Eastern Cooperative Oncology Group

- FDR: false-discovery rate

- HR: Hazard ratio

- IMWG: International Myeloma Working Group

- ISS: International Staging System

- MM: multiple myeloma

- MMRF: Multiple Myeloma Research Foundation

- NDMM: newly diagnosed multiple myeloma

- OS: overall survival

- PFS: progression-free survival

- R-ISS: revised International Staging System

\section{Declarations}

\section{- Ethics approval and consent to participate:}

Not applicable.

\section{- Consent for publication:}

Not applicable.

\section{- Avaibility of data and material:}


The data that support the findings of this study are available from MMRF but restrictions apply to the availability of these data, which were used under license for the current study, and so are not publicly available. Data are however available from the authors upon reasonable request and with permission of MMRF.

\section{- Competing interests:}

M.V.M. receives honoraria and speakers' bureau compensation from Janssen and Celgene, Onyx, Takeda, Novartis, and Bristol-Myers Squibb, unconnected with the submitted work. N.C.G. receives honoraria from Janssen that are unconnected with the work presented here. All the other authors declare no competing financial interests.

\section{- Funding:}

This work was supported by grants from the Instituto de Salud Carlos III, cofinanced by European Union FEDER funds (grants PI16/01074 and PI19/00674) (N.C.G., E.A.R., I.J.C.B., L.A.C.), the Asociación Española Contra el Cáncer (grant PROYE20047GUTI) (N.C.G., C.D.R., E.A.R., I.J.C.B., L.A.C.) and the Gerencia Regional de Salud, Junta de Castilla y León (grants GRS 2058/A/19 and GRS 2331/A/21) (N.C.G., C.D.R., E.A.R., I.J.C.B., L.A.C.). C.D.R, E.A.R. and I.J.C.B. were supported by the Asociación Española Contra el Cáncer (CLJUN18010DERA), the Consejería de Educación de Castilla y León and the Instituto de Salud Carlos III (PFIS-2020: FI20/00226), respectively.

\section{- Authors" contributions:}

C.D.R. carried out the statistical analysis, helped design the study and wrote the manuscript; E.A.R. helped with the statistical analysis; I.J.C.B. helped prepare the figures; M.V.M. helped with data interpretation; L.A.C. designed the research, performed the bioinformatic analysis, created the figures and wrote the manuscript; N.C.G. conceived the idea and helped design the research, supervised the entire study, wrote the manuscript and provided funding; and all authors reviewed and approved the manuscript. *N.C.G. and L.A.C. contributed equally to this work.

\section{- Ackowledgements:}

The authors thank the MMRF for access to the CoMMpass data set. The authors would also like to thank Phil Mason for his help in reviewing the English language of the manuscript.

\section{- Author's information (optional):}


C.D.R is a PhD candidate at Salamanca University (Spain), and this work is submitted in partial fulfillment of the requirement for the $\mathrm{PhD}$.

\section{FOOTNOTES:}

Not applicable.

\section{References}

1. Lohr JG, Stojanov P, Carter SL, Cruz-Gordillo P, Lawrence MS, Auclair D, et al. Widespread genetic heterogeneity in multiple myeloma: implications for targeted therapy. Cancer Cell. 2014 Jan 13;25(1):91-101.

2. Corre J, Perrot A, Caillot D, Belhadj K, Hulin C, Leleu X, et al. del(17p) without TP53 mutation confers a poor prognosis in intensively treated newly diagnosed patients with multiple myeloma. Blood. 2021;137(9):1192-1195.

3. Walker BA, Boyle EM, Wardell CP, Murison A, Begum DB, Dahir NM, et al. Mutational Spectrum, Copy Number Changes, and Outcome: Results of a Sequencing Study of Patients With Newly Diagnosed Myeloma. J Clin Oncol. 2015;33(33):3911-20.

4. Walker BA, Mavrommatis K, Wardell CP, Ashby TC, Bauer M, Davies FE, et al. Identification of novel mutational drivers reveals oncogene dependencies in multiple myeloma. Blood. 2018;132(6):587597.

5. Lodé L, Eveillard M, Trichet V, Soussi T, Wuillème S, Richebourg S, et al. Mutations in TP53 are exclusively associated with del(17p) in multiple myeloma. Haematologica. 2010;95(11):1973-6.

6. Walker BA, Mavrommatis K, Wardell CP, Ashby TC, Bauer M, Davies F, et al. A high-risk, Double-Hit, group of newly diagnosed myeloma identified by genomic analysis. Leukemia. 2019;33(1):159-170.

7. Teoh PJ, Chung TH, Sebastian S, Choo SN, Yan J, Ng SB, et al. p53 haploinsufficiency and functional abnormalities in multiple myeloma. Leukemia. 2014;28(10):2066-74.

8. Ziccheddu B, Da Vià MC, Lionetti M, Maeda A, Morlupi S, Dugo M, et a. Functional Impact of Genomic Complexity on the Transcriptome of Multiple Myeloma. Clin Cancer Res. 2021;27(23):64796490.

9. Avet-Loiseau H. Ultra high-risk myeloma. Hematology Am Soc Hematol Educ Program. 2010;2010:489-93.

10. Miller C, Yesil J, Derome M, Donnelly A, Marrian J, McBride K, et al. A comparison of clinical FISH and sequencing based FISH estimates in multiple myeloma: an MMRF CoMMpass analysis. Blood. 2016;128(22):374.

11. Ennishi D, Jiang A, Boyle M, Collinge B, Grande BM, Ben-Neriah S, et al. Double-hit gene expression signature defines a distinct subgroup of germinal center B-cell-like diffuse large B-cell lymphoma. J Clin Oncol. 2019;37(3):190-201. 
12. Edgar R, Domrachev M, Lash AE. Gene Expression Omnibus: NCBI gene expression and hybridization array data repository. Nucleic Acids Res. 2002;30(1):207-10.

13. Danziger SA, McConnell M, Gockley J, Young MH, Rosenthal A, Schmitz F, et al. Bone marrow microenvironments that contribute to patient outcomes in newly diagnosed multiple myeloma: A cohort study of patients in the Total Therapy clinical trials. PLoS Med. 2020;17(11):e1003323.

14. Dai M, Wang P, Boyd AD, Kostov G, Athey B, Jones EG, et al. Evolving gene/transcript definitions significantly alter the interpretation of GeneChip data. Nucleic Acids Res. 2005;33(20):e175.

15. Zhan F, Huang Y, Colla S, Stewart JP, Hanamura I, Gupta S, et al. The molecular classification of multiple myeloma. Blood. 2006;108(6):2020-8.

16. Liu H, Bebu I, Li X. Microarray probes and probe sets. Front Biosci (Elite Ed). 2010 Jan 1;2:325-38.

17. Szklarczyk D, Gable AL, Nastou KC, Lyon D, Kirsch R, Pyysalo S, et al.The STRING database in 2021: customizable protein-protein networks, and functional characterization of user-uploaded gene/measurement sets. Nucleic Acids Res. 2021 Jan 8;49(D1):D605-D612.

18. Xu-Monette ZY, Medeiros LJ, Li Y, Orlowski RZ, Andreeff M, Bueso-Ramos CE, et al.Dysfunction of the TP53 tumor suppressor gene in lymphoid malignancies. Blood. 2012;119(16):3668-83.

19. Miller LD, Smeds J, George J, Vega VB, Vergara L, Ploner A, et al. An expression signature for p53 status in human breast cancer predicts mutation status, transcriptional effects, and patient survival. Proc Natl Acad Sci U S A. 2005;102(38):13550-5.

20. Zhao Y, Varn FS, Cai G, Xiao F, Amos Cl, Cheng C. A P53-Deficiency Gene Signature Predicts Recurrence Risk of Patients with Early-Stage Lung Adenocarcinoma. Cancer Epidemiol Biomarkers Prev. 2018 Jan;27(1):86-95.

21. Xiong W, Wu X, Starnes S, Johnson SK, Haessler J, Wang S, et al. An analysis of the clinical and biologic significance of TP53 loss and the identification of potential novel transcriptional targets of TP53 in multiple myeloma. Blood. 2008;112(10):4235-46.

22. Greipp PR, San Miguel J, Durie BG, Crowley JJ, Barlogie B, Bladé J, et al. International staging system for multiple myeloma. J Clin Oncol. 2005;23(15):3412-20.

23. Chng WJ, Dispenzieri A, Chim CS, Fonseca R, Goldschmidt H, Lentzsch S, et al; International Myeloma Working Group. IMWG consensus on risk stratification in multiple myeloma. Leukemia. 2014 Feb;28(2):269-77.

24. Palumbo A, Avet-Loiseau H, Oliva S, Lokhorst HM, Goldschmidt H, Rosinol L, et al.Revised International Staging System for multiple myeloma: a report from International Myeloma Working Group. J Clin Oncol. 2015;33(26):2863-9.

25. Skerget S, Penaherrera D, Chari A, Jagannath S, Siegel DS, Vij R et al. Genomic basis of multiple myeloma subtypes from the MMRF CoMMpass study. Preprint athttps://www.medrxiv.org/content/10.1101/2021.08.02.21261211v1 (2021)

26. Thakurta A, Ortiz M, Blecua P, Towfic F, Corre J, Serbina NV, et al. High subclonal fraction of 17p deletion is associated with poor prognosis in multiple myeloma. Blood. 2019;133(11):1217-1221. 
27. Herrero AB, Rojas EA, Misiewicz-Krzeminska I, Krzeminski P, Gutiérrez NC. Molecular mechanisms of p53 deregulation in cancer: an overview in multiple myeloma. Int J Mol Sci. 2016;17(12):2003.

28. Wasylishen AR, Lozano G. Attenuating the p53 pathway in human cancers: many means to the same end. Cold Spring Harb Perspect Med. 2016;6(8):a026211.

\section{Figures}

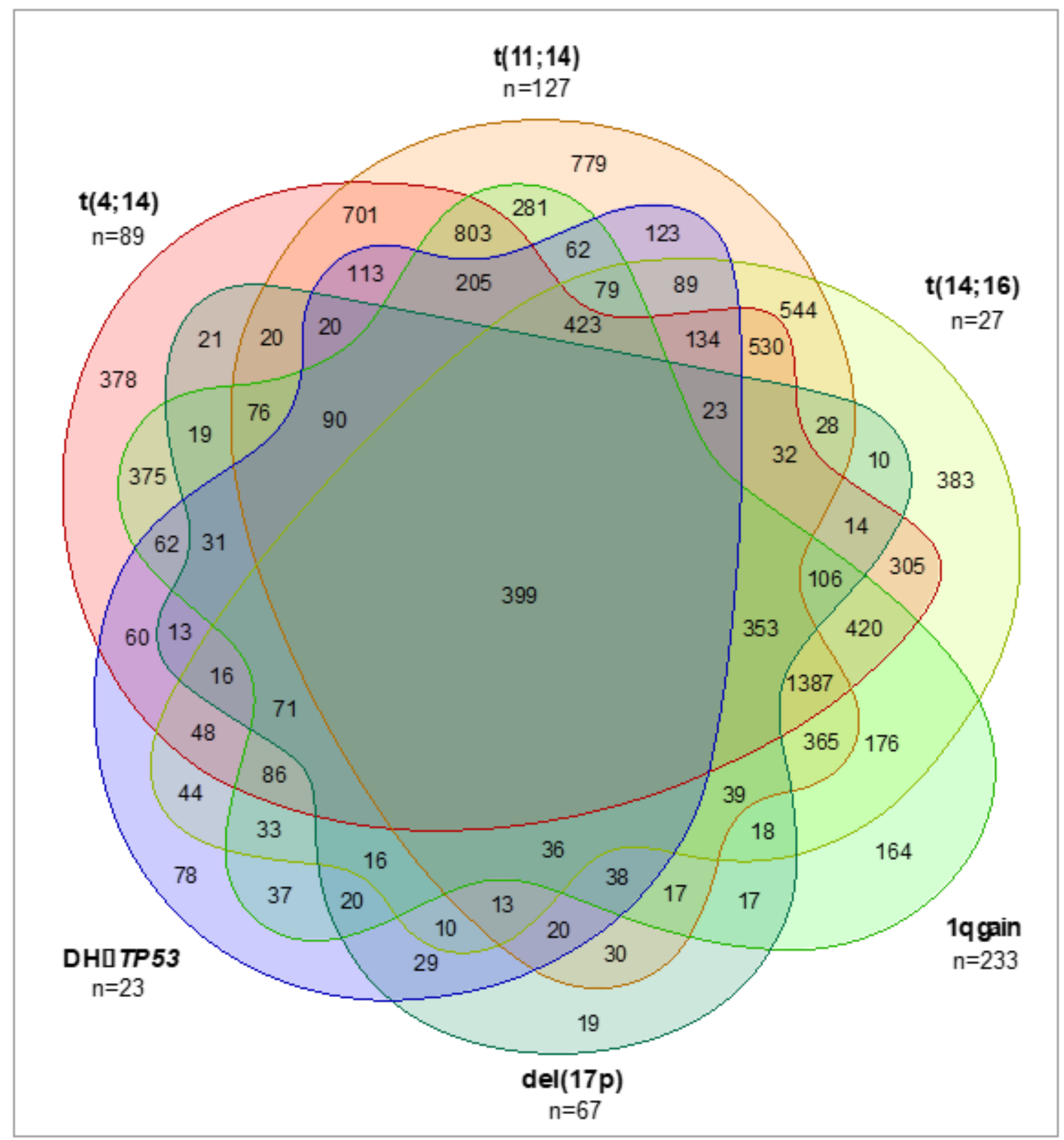

\section{Figure 1}

Expression signature of TP53 biallelic inactivation and score generation. Venn diagram indicating the number of differentially expressed genes in the six groups of NDMM defined according to cytogenetic 
abnormalities and TP53 mutations. The non-overlapping numbers indicate the differentially expressed genes unique to each group.
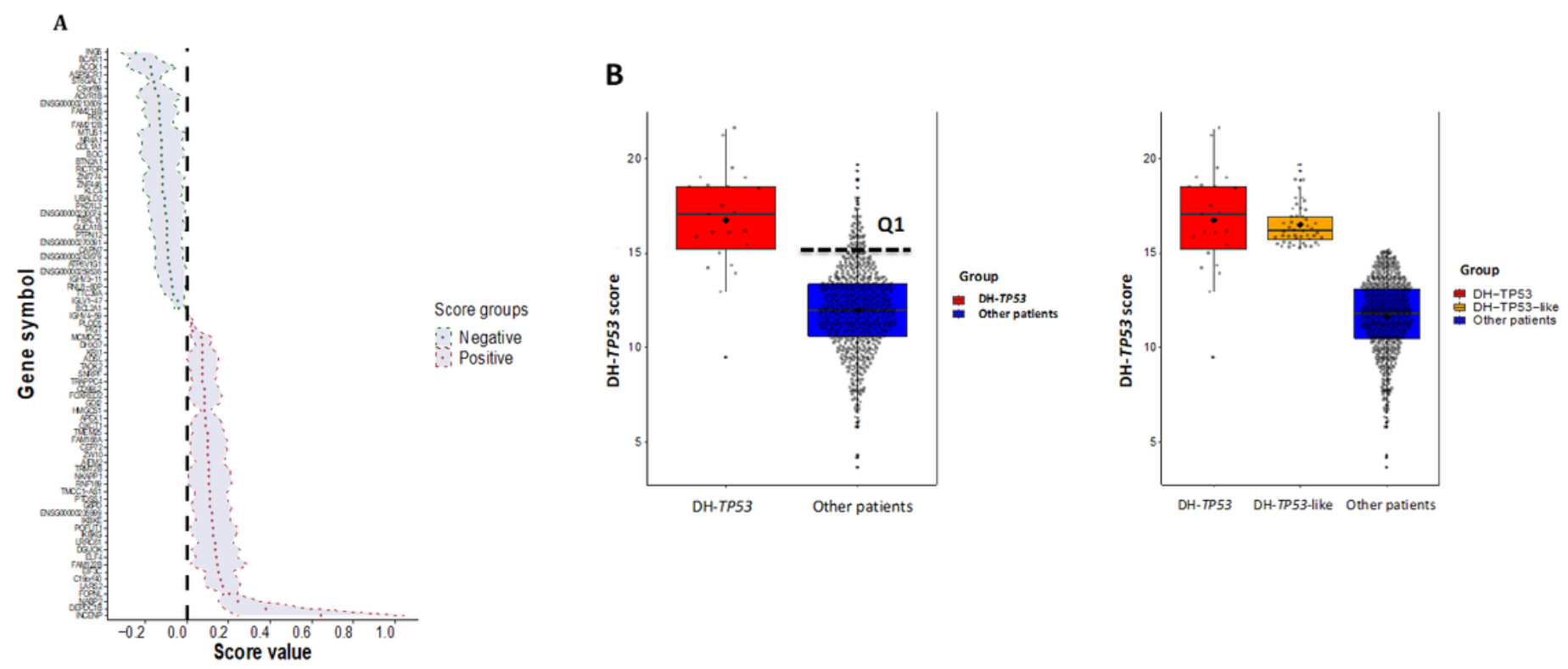

Figure 2

DH-TP53 score generation. (A) The 78 differentially expressed genes in patients harboring TP53 biallelic inactivation are represented according to their scores. (B) Representation of scores for each patient. The first quartile (Q1) of the score in the DH-TP53 patients is indicated. This is applied to the non-DH-TP53 group, giving rise to the DH-TP53-like group, indicated in yellow.
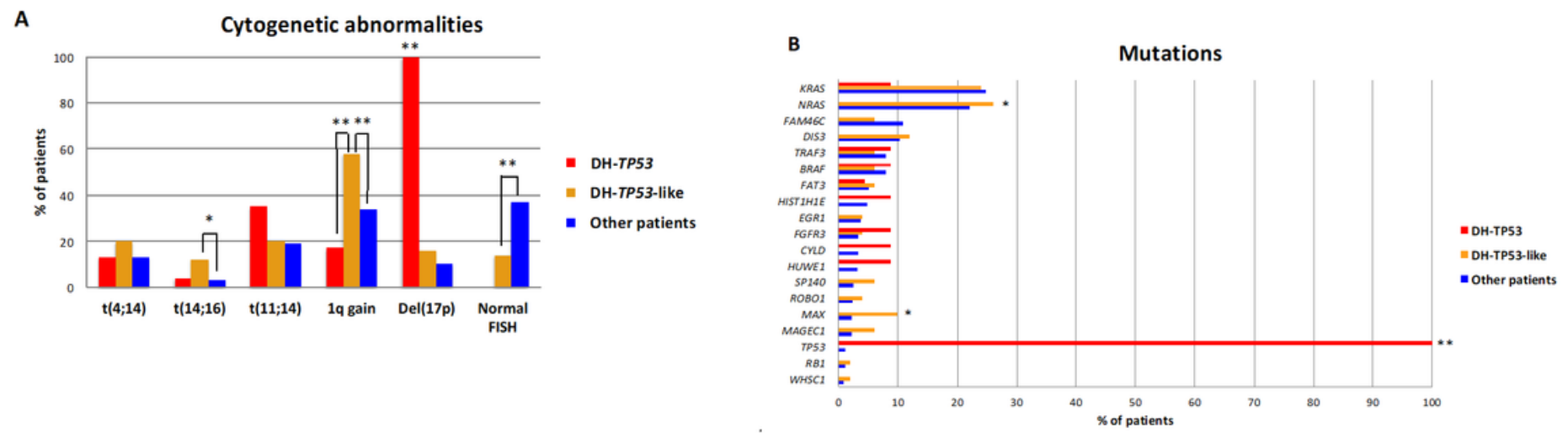

Figure 3 
Genetic characteristics of the DH-TP53 and DH-TP53-like groups. (A) Association between the most frequent cytogenetic abnormalities in MM and the three groups defined by the score and DH-TP53 condition. (B) Association between the most frequently mutated genes in $\mathrm{MM}$ and the three groups defined by the score and DH-TP53 condition. Only non-synonymous mutations are shown. ${ }^{*} p<0.05$, ** $p$ $<0.01$. Note that the proportion of $\operatorname{del}(17 p)$ and TP53 mutations is $100 \%$ in DH-TP53 group by definition.

A

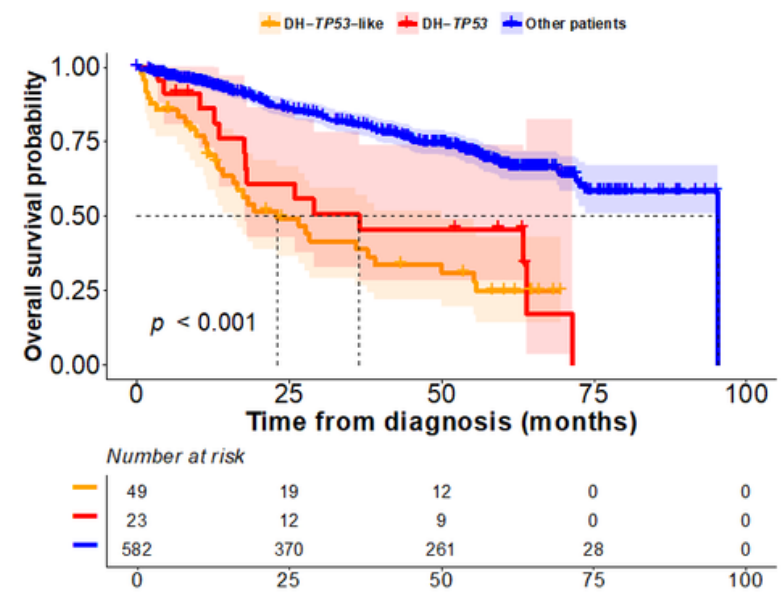

B

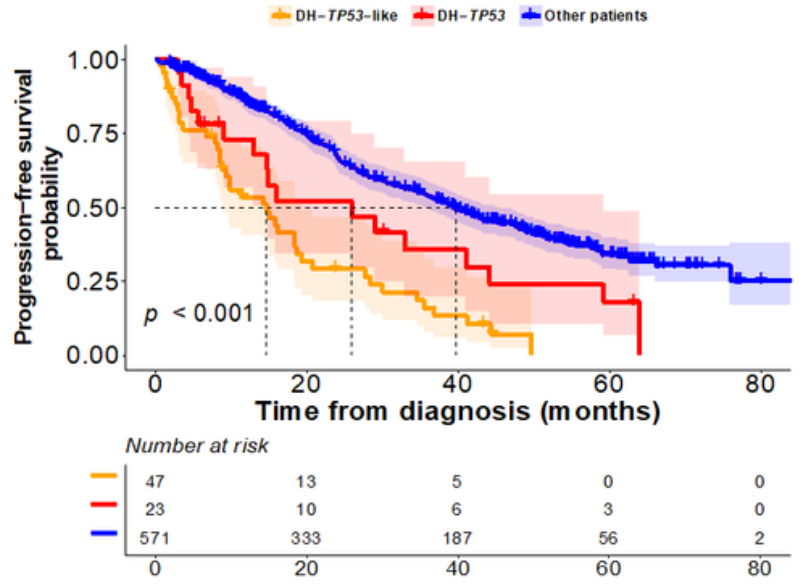

Figure 4

Prognosis of the DH-TP53 and DH-TP53-like groups. Probability of overall survival (A) and progressionfree survival (B), according to the groups defined by the score and DH-TP53 condition. The log-rank (Mantel-Cox) test $p$ values are shown. 

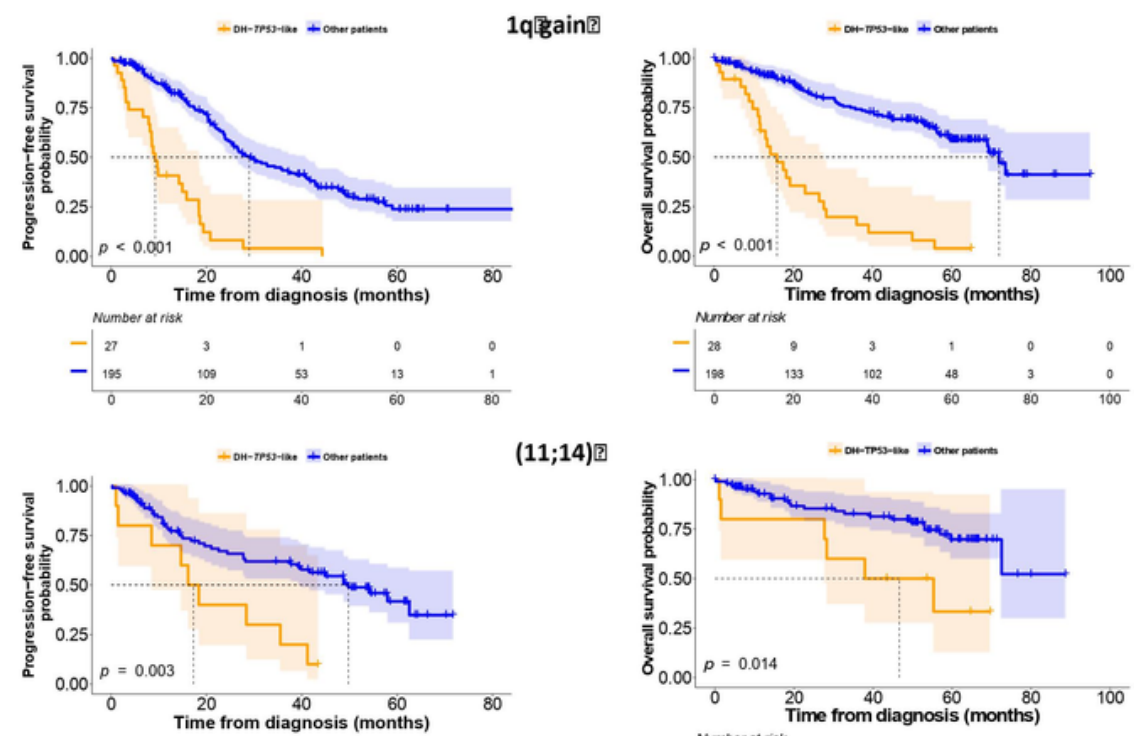

$(11 ; 14)$ 圆
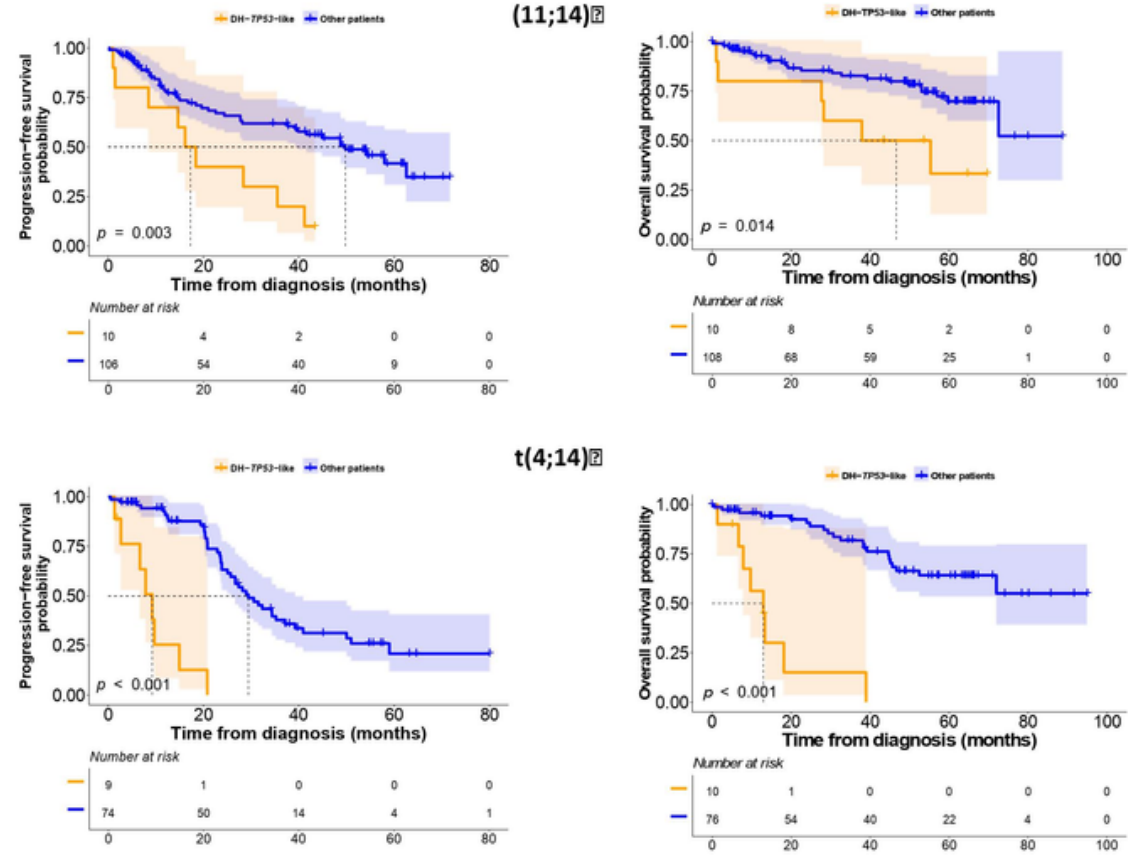

$t(4 ; 14)$ ]
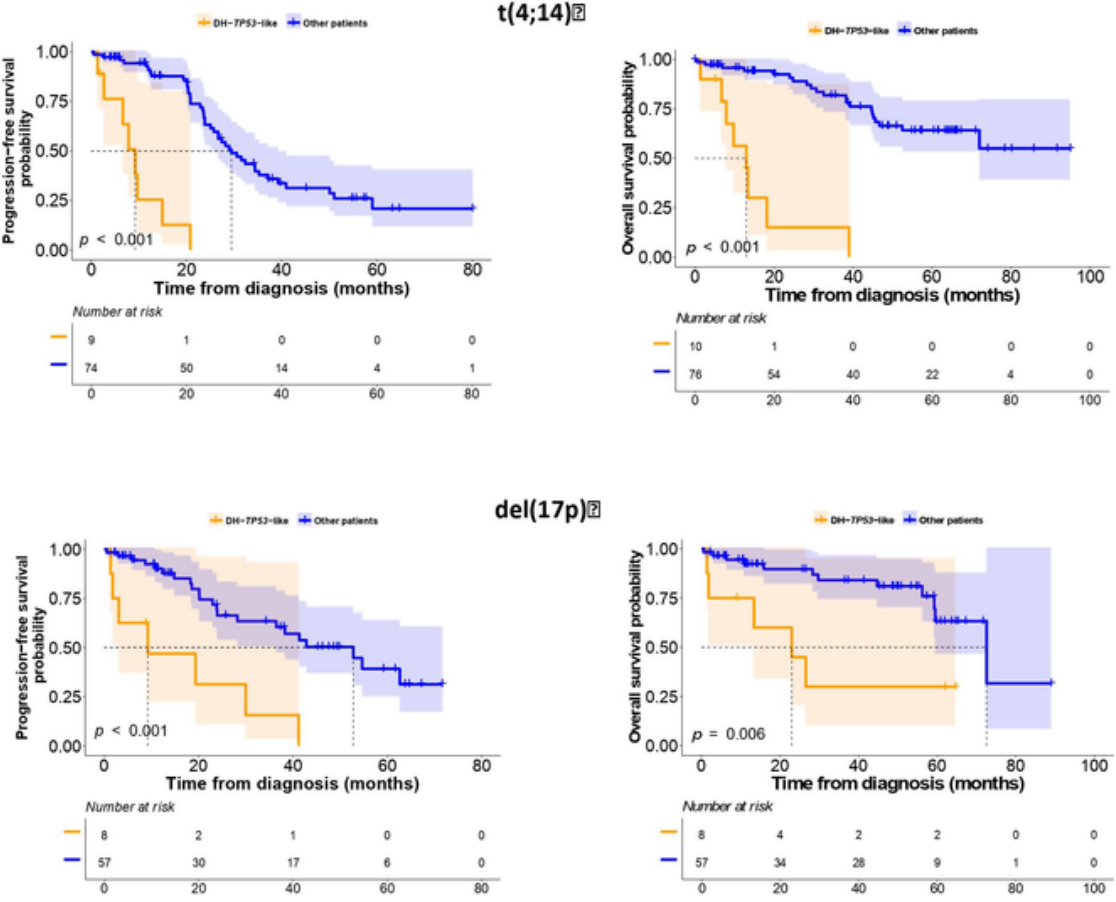

Figure 5

Prognosis of cytogenetic abnormalities according to whether they belong to DH-TP53-like group or not. Probability of progression-free survival and overall survival in the group of patients with 1q gain, $t(11 ; 14)$, $\mathrm{t}(4 ; 14)$ and del(17p). The log-rank (Mantel-Cox) test $p$ values are shown. 
A
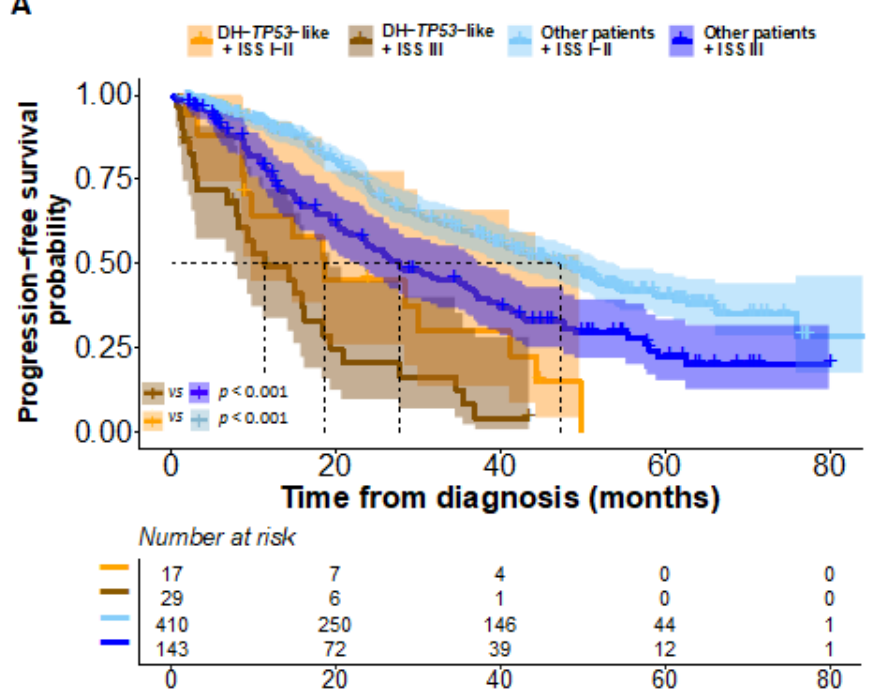

B

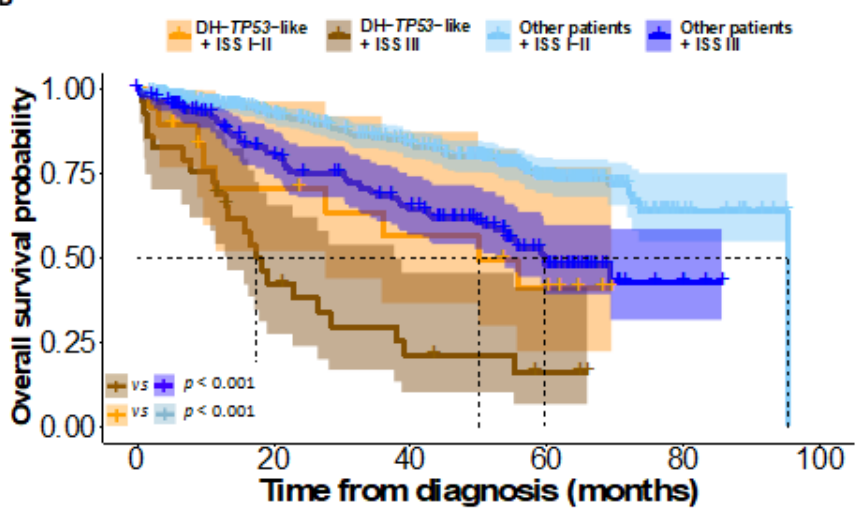

Number at risk

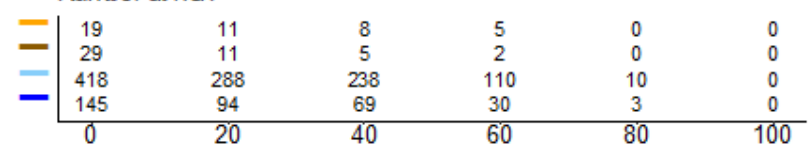

Figure 6

Prognosis of the DH-TP53-like group according to ISS index. Probability of progression-free survival (A) and overall survival (B) in the DH-TP53-like group and the other patients (excluding DH-TP53 patients) when combined with ISS stage. The log-rank (Mantel-Cox) test $p$ values are shown.

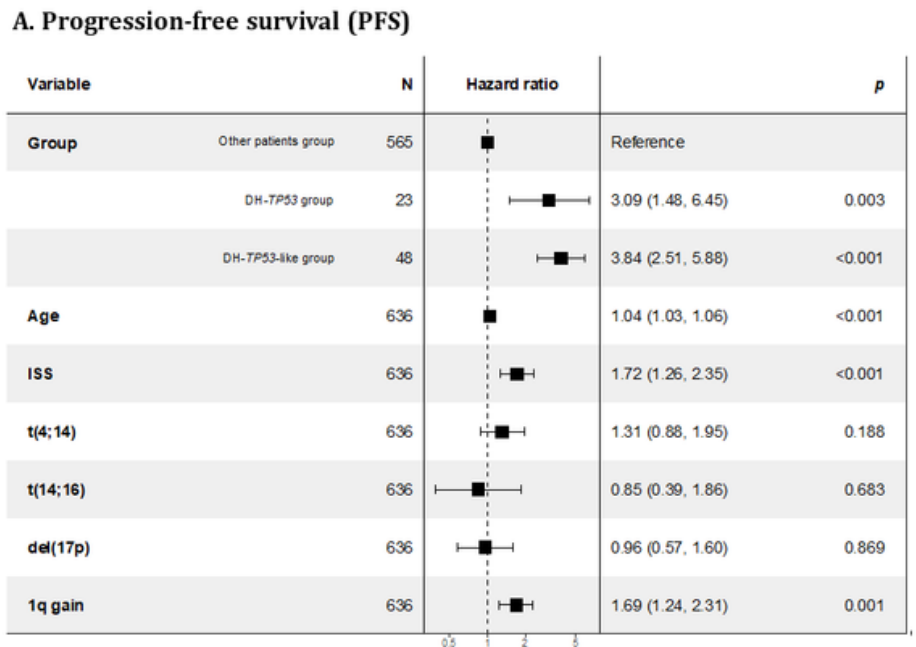

B. Overall survival (OS)

\begin{tabular}{|c|c|c|c|c|c|}
\hline Variable & & $\mathrm{N}$ & Hazard ratio & & $\rho$ \\
\hline \multirow[t]{3}{*}{ Group } & Other pastens group & 553 & 中 & Reference & \\
\hline & DH.TP53 grovp & 23 & $\longrightarrow$ & $1.98(1.07,3.66)$ & 0.030 \\
\hline & DH-TPS3Ake group & 46 & $\mapsto$ & $3.32(2.31,4.77)$ & $<0.001$ \\
\hline Age & & 622 & i & $1.03(1.02,1.04)$ & $<0.001$ \\
\hline ISS & & 622 & 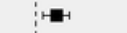 & $1.42(1.12,1.81)$ & 0.004 \\
\hline$t(4 ; 14)$ & & 622 & $H$ & $1.39(1.03,1.88)$ & 0.033 \\
\hline$t(14 ; 16)$ & & 622 & 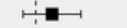 & $1.32(0.79,2.20)$ & 0.289 \\
\hline del(17p) & & 622 & $\mapsto$ & $0.92(0.62,1.37)$ & 0.679 \\
\hline 1q gain & & 622 & $=$ & $1.36(1.08,1.72)$ & 0.010 \\
\hline
\end{tabular}

\section{Figure 7}

Multivariable analysis of OS and PFS. Forest plot of multivariable Cox proportional-hazards models for progression-free survival (A) and overall survival (B) with the hazard ratio for each of the factors included: groups defined by DH-TP53 score, age (as a continuous variable [years]), ISS III vs I/II, presence of $t(4 ; 14)$, $\mathrm{t}(14 ; 16)$, del(17p) and 1q gain. $p$ values for each factor are shown. 


\section{Supplementary Files}

This is a list of supplementary files associated with this preprint. Click to download.

- JHOAdditionalfile1.docx

- JHOAdditionalfile2.docx

- JHOAdditionalfile3.docx 